relatively poor regardless of the surgical procedures performed and adjuvant therapies given.

Disclosures No potential conflict of interest to declare.

\section{A SYSTEMATIC REVIEW TO IDENTIFY AND ASSESS THE MENTAL HEALTH SEQUALAE AMONGST WOMEN WITH ENDOMETRIOSIS WITH OR WITHOUT CHRONIC PELVIC PAIN (THE ELEMI PROJECT)}

'Dharani Hapangama, ${ }^{2}$ Gayathri Delanerolle, ${ }^{3}$ Peter Phiri, ${ }^{2}$ Rema Ramakrishnan, ${ }^{4}$ Ashish Shetty, ${ }^{5}$ Trusha Kotari, ${ }^{6}$ Katherine Barnard, ${ }^{7}$ Molola Oyewole, ${ }^{8}$ Damien Longson, ${ }^{9}$ Vanessa Raymont. 'University of Liverpool; Liverpool Women's Hospital NHS Trust; ${ }^{2}$ University of Oxford; ${ }^{3}$ Southern Health NHS Trust; University of Southampton; ${ }^{4}$ University College London NHS Foundation Trust; University College London; ${ }^{5}$ Liverpool Women's Hospital NHS Trust; ${ }^{6}$ University of Southampton; 'University College London NHS Foundation Trust; ${ }^{8}$ University of Manchester; ${ }^{9}$ University of Oxford; Oxford Health NHS Foundation Trust

\subsection{6/ijgc-2020-ESG0.93}

Introduction/Background Endometriosis is a complex, chronic gynaecological condition impacting approximately 176 million women globally. It is associated with symptoms such as chronic pelvic pain (CPP), dysmenorrhoea, menorrhagia, sexual dysfunction and infertility. During several points in the lifecycle of this chronic disease, women bear the consequent burden of mental health $(\mathrm{MH})$ difficulties, due to the complex symptomatology and comorbidities of endometriosis. For example, delayed diagnosis (the average time to diagnose being 7-8 years), undergoing often repeated

\section{Forest plot of pooled prevalence of anxiety}

\begin{tabular}{lll} 
Author & $\begin{array}{l}\text { Percent } \\
(95 \% \mathrm{Cl})\end{array}$ \\
\hline Cavaggioni, 2014 & $30.00(20.26,41.28)$ \\
De Graaff, 2016 & $32.51(26.66,38.79)$ \\
Friedl, 2015 & $17.74(9.20,29.53)$ \\
Laganà, 2015 & $40.96(33.40,48.85)$ \\
Sepulcri, 2009 & $28.85(20.38,38.55)$ \\
Simoens, 2012 & $35.97(32.85,39.19)$ \\
Overall (I $=72.7 \%, \mathrm{p}<0.001)$ & $31.80(26.51,37.09)$ \\
& 20 & 100 \\
\hline A & Percent
\end{tabular}

Forest plot of pooled prevalence of depression

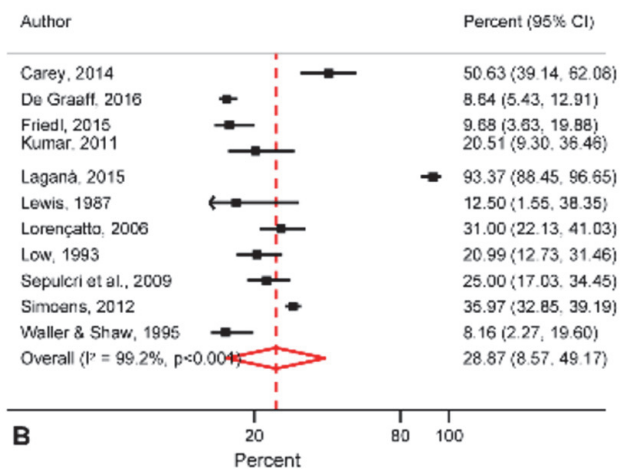

Abstract 369 Figure 1 excision surgeries, and difficulties with subfertility and sexual activities, and suffering with long-term CPP and analgesic use, can all negatively influence $\mathrm{MH}$ and can have significant impact on the psychological, sexual, relationship and social functioning of affected women. Therefore, a systematic review was conducted as part of the evidence synthesis phase of the ELEMI project to identify and assess this complex relationship with a view to report on any knowledge and clinical practice gaps.

Methodology The systematic protocol was published in PROSPERO (CRD42020181495). MeSH terms developed include Endometriosis, Depression, Anxiety, Low mood, Psychiatry comorbidity, Women's health and CPP. All studies and material published between January 1980 to June 2020 in English and participants were included.

Results Out of 28 studies included in the systematic review, 17 were included in the meta-analysis (anxiety: 6, chronic pelvic pain: 3, depression: 11, and dyspareunia: 3) which described the prevalence and extent of $\mathrm{MH}$ symptoms in endometriosis and/or CPP. The pooled prevalence for anxiety was found to be $31.8 \%$ (95\% CI: $26.5 \%$ - 37.1\%), whilst for depression 28.9\% (95\%CI: 8.6\%-49.2\%). Pooled prevalence for CPP was high at 57.2\% (95\%CI: $7.0 \%$, $107.4 \%)$ and pooled estimate of mean SF-MPQ for chronic pain to be 13.09 (95\%CI: 7.13, 19.05). Computed prevalence for dyspareunia was also identified to be high (prevalence: $54.9 \%$, 95\% CI: 43.9\%, 65.9\%). The narrative analysis showed depression and anxiety to be the most commonly reported $\mathrm{MH}$ symptoms. None of the papers indicated if these had received a clinical diagnosis or were

\section{Forest plot of pooled prevalence of chronic pelvic pain}

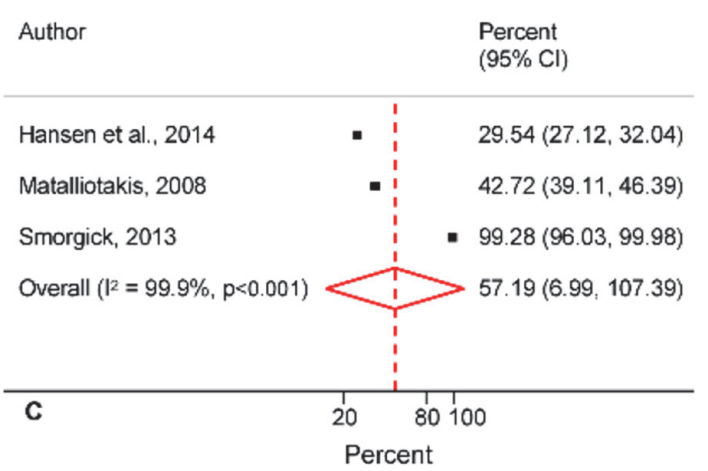

Forest plot of pooled prevalence of dyspareunia

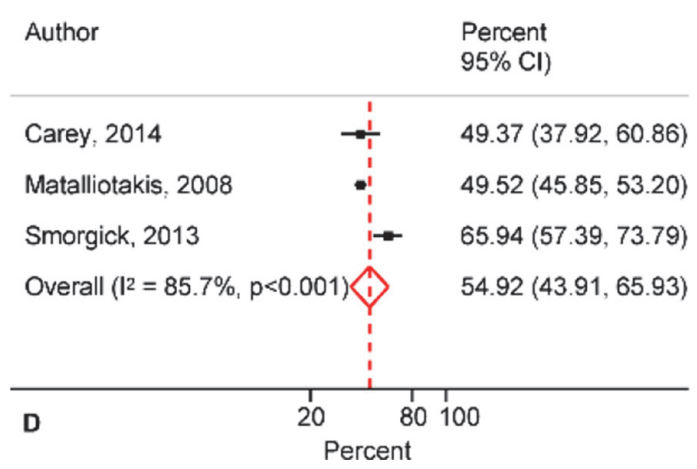


being treated. Generic $\mathrm{MH}$ assessments were used in all samples. Thus, whilst it is vital to identify the reporting of these symptoms, their clinical significance is yet to be comprehensively ascertained, and further exploratory evidence is required (figure 1 ).

Conclusion Limited research is currently available to evaluate the $\mathrm{MH}$ sequalae of endometriosis. Further comprehensive research is required to fully assess and treat the $\mathrm{MH}$ associated endometriosis patient reported outcomes.

Disclosures Nothing to declare

\section{SAFETY OF ONCOLOGIC SURGERY WITHOUT PREOPERATIVE COVID-19 TESTING IN ASYMPTOMATIC PATIENTS}

${ }^{1}$ Nazlı Orhan, ${ }^{2}$ Utku Akgor, ${ }^{1}$ Nejat Ozgul, ${ }^{1}$ Mehmet Coskun Salman, ${ }^{1}$ Murat Gultekin. ${ }^{1}$ Hacettepe University Faculty of Medicine; Department of Obstetrics and Gynaecology; ${ }^{2}$ Hacettepe University Faculty of Medicine; Department of Gynecological Oncology; Department of Obstetrics and Gynaecology

\subsection{6/ijgc-2020-ESG0.94}

Introduction/Background Novel Coronavirus Disease (COVID19) pandemic has a significant impact on healthcare services. Non-emergent surgeries are being restricted since medical facilities are mostly occupied by or reserved for COVID-19 patients. However, postponing oncologic surgeries may have significant effects on survival. Also, many reports were published regarding the safety of oncologic surgeries during the pandemic. In this study, we aimed to evaluate the safety of oncologic surgeries without preoperative COVID-19 testing in asymptomatic patients with gynaecological cancers when preoperative COVID-19 testing was not mandatory.

Methodology Patients with gynaecological cancers who were operated between March 11 to June 11 without preoperative COVID-19 testing at Hacettepe University Faculty of Medicine, Department of Obstetrics and Gynaecology were identified. These patients were followed up 6 weeks after surgery to evaluate COVID-19 related morbidity or mortality.

Results The study group consisted of 30 patients. Of these, 17 had endometrial cancer, 9 had ovarian cancer, 3 had cervical, and 1 had vulvar cancer. Mean age of patients was 58 years. Twenty-two patients $(73.3 \%)$ had co-morbidities and among those $3(10.0 \%)$ had pulmonary disease. Five patients $(16.7 \%)$ were followed up in the intensive care unit postoperatively based on decision of attending anesthesiologists. Despite venous thromboembolism prophylaxis, one patient $(3.3 \%)$ with ovarian cancer developed pulmonary embolism who was tested negative for COVID-19. This patient developed acute abdomen on 20th postoperative day and was subjected to relaparotomy, but she died of cardiac arrest 3 days later. No significant morbidity or mortality was observed in remaining 29 patients $(96.7 \%)$.

Conclusion During the COVID-19 pandemic, risk of surgical morbidity or mortality is not increased and oncologic surgeries may safely be performed without routine COVID-19 testing in asymptomatic cases even if they have co-morbidities. However, adequate infrastructure is crucial since postoperative intensive care unit admission is required for a significant proportion of patients.

Disclosures No potential conflict of interest to declare.

\section{ADRENAL INCIDENTALOMAS IN GYNAECOLOGY ONCOLOGY SURGICAL PATIENTS: TIMELY INVESTIGATION AND MANAGEMENT AVOIDING UNDUE DELAYS TO ONCOLOGICAL SURGERY}

Marie-Therese Grant, Dhivya Chandrasekaran, Daniel Moganstein, Aslam Sohaib, Marielle Nobbenhuis. Royal Marsden Hospital; London

\subsection{6/ijgc-2020-ESGO.95}

Introduction/Background The prevalence of incidental adrenal nodules is $4 \%$. Although the majority of incidental adrenal nodules are benign (85\%) and non-functional, malignant and hormone-secreting nodules (excess cortisol, aldosterone or catecholamines) require timely investigation and management. Currently, there is limited literature on their management in the pre-operative oncology setting and further investigation is time consuming and often causes significant delays to the planned gynaecological oncology surgical treatment.

Methodology Retrospective single cohort observational study undertaken at a tertiary cancer centre in UK. Patients undergoing major gynaecology oncology surgery who had incidental adrenal pathology reported on pre-operative imaging over a one year period (July 2019 - July 2020) were identified. Patient demographics, endocrine investigations and delays in treatment pathways were reported using descriptive statistics.

Results Incidental finding of adrenal pathology were reported in 9 of the 346 cases (2.6\%). Patient age ranged from 30-73 years old. The primary sites of malignancy included ovarian (4/9), endometrial (4/9) and cervical (1/9). All adrenal pathology was identified on preoperative CT; the reports did not categorise these into benign or malignant/functioning. The size of the adrenal pathologies ranged from $11 \mathrm{~mm} \mathrm{-} 70 \mathrm{~mm}$ (median $=26 \mathrm{~mm})$. PET CT was used to further evaluate an adrenal nodule (1/9).

In those cases that had further investigations, initial tests included both urinary and plasma metanephrines $1 / 9$, plasma metanephrines (2/9) and urinary metanephrines (5/9). Additional tests were undertaken in two cases; a dexamethasone suppression test (1/9) and serum renin/aldosterone (2/9). From these, 2/9 had abnormal urinary metanephrines. 1/9 was a false positive result and the other had endocrinology review for a raised methotyramine only which was not felt to be clinically significant. An adrenal malignancy or phaemochromocytoma was not identified within the cohort. (2/9) had no investigations or endocrinology input. The planned gynaecological surgical date was postponed in $4 / 9$ cases to allow for investigation of the adrenal pathology to rule out a functioning or malignant lesion. The delay to surgery ranged 0-56 days (median 21 days). There was no progression of disease or change to the original management plan as a result of the delay.

Conclusion There is heterogeneity in the investigation and management of incidental adrenal pathology and significant delays to the surgical treatment of the patients' primary gynaecology malignancy. We recommend adopting the European Society of Endocrinology guidelines to develop a local pathway with input from our endocrinology and radiology colleagues to ensure these patients are investigated in a timely and standardised manner and avoid undue delays to surgical treatment for the patient's gynaecological malignancy.

Disclosures None of the authors wish to disclose any potential conflict of interest. 